\title{
Performance Analysis of Closed-Loop MIMO system
}

\author{
M.Raja \\ Department of Electronics and Communication \\ Engineering \\ National Institute of Technology (NIT) \\ Tiruchirappalli, India.
}

\author{
Dr.P.Muthuchidambaranathan \\ Department of Electronics and Communication \\ Engineering \\ National Institute of Technology (NIT) \\ Tiruchirappalli, India.
}

\begin{abstract}
In this paper, it investigate the bit error rate (BER) performance of transmit beamforming using singular value decomposition (SVD) for closed loop multiple-input multiple-output (MIMO) wireless systems with various modulation techniques such as binary phase shift keying (BPSK), quadrature phase-shift keying (QPSK) and 16- quadrature amplitude modulation (16-QAM) along with convolution encoder and viterbi decoder. Beamforming separates the MIMO channel into parallel subchannels. The beamforming vectors used at the transmitter and the receiver can be obtained by the singular value decomposition (SVD) of the MIMO channel. Signals are transmitting in the direction of the eigenvector corresponding to the largest eigen value of the channel. The transmit beamforming is performed by multiplying the input symbols with beamforming vector (i.e.) unitary matrix and the precoded symbols are transmitted over rayleigh fading channel. At the receiving end the transmitted signals are obtained by performing the receiver shaping by multiplying the received signal with conjugate transpose of the unitary matrix. Furthermore, derive an expression for a capacity of MIMO system and derive expressions for average BER for BPSK and average symbol error rate (SER) for M-QAM. Simulation results displays the diversity performance of the single beamforming when the three modulations are used separately and it shows the proposed SVD-based beamforming with convolution encoder yield the better performance when compare to the other beamforming method.
\end{abstract}

\section{Keywords}

SVD, Rayleigh Fading Channel, BER, Transmit-Beamforming, Closed-Loop MIMO, BPSK, 16-QAM, QPSK.

\section{INTRODUCTION}

Multiple-Input Multiple-Output (MIMO) systems have recently developed as one of the most important technical breakthroughs for next generation communication systems. The use of multiple antennas at both transmitter and receiver in wireless communication links has been shown to be capable of achieving extraordinary bit rates without incurring any penalty in power or bandwidth [1] [2].The capacity analysis of MIMO systems has shown significant gains over single-input single-output (SISO) systems [2]. Depending on the channel condition, the first generation MIMO technique aims at achieving a higher data rate, such as spatial multiplexing [3], or a higher diversity, such as space-time coding [4]. These techniques do not require the knowledge of channel state information (CSI) at the transmitter. In [5] and [6], it is suggested that an additional performance gain can be extracted from multiple antennas in the presence of channel state information at the transmitter. When perfect CSI is available at both ends, beamforming is used to maximize the SNR at the receiver. Beamforming separates the MIMO channel into parallel independent subchannels. When the subchannel with the largest gain is used for transmission, the technique is called single beamforming [7]. MIMO systems can also be used to enhance the throughput of wireless systems [8]. In the context of feedback systems, more than one subchannel can be used to improve the capacity. This technique is called multiple beamforming. Most work on closed-loop MIMO systems has been carried out by performing SVD of the channel transfer matrix. Among linear precoders, the SVD-based beamforming technique combined with a proper power allocation method is shown to be optimum in terms of capacity [9].

In this paper, At the transmitter we propose a transmit precoding approach by multiplying the matrix $V$ to perform transmit beamforming and the precoded symbols are transmitted over the Rayleigh fading channel by $M_{t}$ transmitting antenna, at the receiver faded version of the transmitted symbols which are received by $M_{r}$ receiving antenna and the MRC is used as a combiner and the receiver shaping is performed by multiplying the channel output with matrix $U^{H}$. Here we use the BPSK, QPSK and 16-QAM for modulating the transmit signals and convolution encoder and viterbi decoder are used to archive the reliable data transfer, and we derive the expression for a capacity of MIMO system when SVD is used for channel decomposition. The organization of the paper is as follows: Section II presents a general description of SVD-based transmit beamforming system model. In Section III, derive expressions for average bit error rate (BER) for BPSK and average symbol error rate (SER) for MQAM. In Section III, we drive the expression for capacity of MIMO. Section V shows the simulation results and compares the results of various modulations. Finally, the paper is terminated with conclusions in Section V.

\section{SYSTEM MODEL}

In this section, we present a general description of transmitter and receiver sections of SVD-based transmit beamforming. We consider the MIMO system models with $M_{t}$ transmit antennas and $M_{r}$ receive antennas, as shown in Fig. 1.

Transmission is over a rayleigh fading channel and both the transmitter and receiver are assumed to have perfect knowledge of the channel. The system consists of a beamforming processing at 


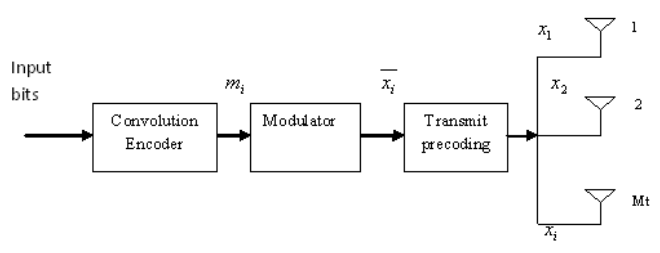

(a)Transmitter

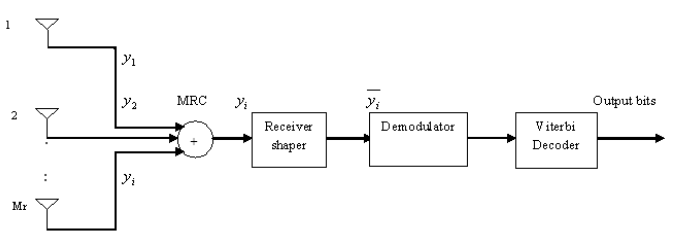

(b) Receiver

Figure 1. Schematic diagram of a transmit beamforming and receive combining scheme with $M_{t}$ transmit and $M_{r}$ receive antennas.

the transmitter and combining processing at the receiver. At the transmitter, the information bits $s=\left\{s_{1}, s_{2} \ldots \ldots s_{k}\right\}$ are encoded with convolution encoder and, the encoded symbols are modulated by either BPSK or QPSK or 16-QAM modulator which is used as symbol mapper, to yield the symbol vector of $\bar{x}=\left\{\overline{x_{1}}, \overline{x_{2}} \ldots \ldots . . \overline{x_{k}}\right\}$, where $k$ is the number of transmitted symbols.

This data symbol $x$ is applied to the transmit precoding and multiplied by matrix $V$ to perform transmit beamforming, and the precoded symbols $x=\left\{x_{1}, x_{2} \ldots \ldots x_{k}\right\}$ are transmitted over rayleigh fading channel. We assume that the elements of the MIMO channel matrix are obtained from an independent and identical distribution (i.i.d) complex Gaussian distribution with zero mean and unit variance. If $x$ is the $1 \times M_{t}$ vector containing the symbols to be transmitted, $H$ is the channel matrix of size $M_{t} \times M_{r}$ and $n$ a vector of additive white gaussian noise (AWGN) on the receiving antenna of size $l \times M_{r}$, the vector of received symbol can be expressed as

$$
y=H x+n,
$$

At the receiver, the transmitted symbol $y=\left\{\begin{array}{llll}y_{1}, y_{2} & \ldots & y_{k}\end{array}\right\}$ are received with addition of channel noise $n$ and the combining process to be performed for receiving symbols with MRC, receiver shaping is performed at the receiver by multiplying the channel output $y$ with to produce $\bar{y}=\left\{\overline{y_{1}}, \overline{y_{2}} \ldots \ldots . \overline{y_{k}}\right\}$ and finally, the decoding and demapping operation is performed to produce the output.

\subsection{SVD-based beamforming overview}

Beamforming is implemented by multiplying the symbols with appropriate beamforming vectors, both on transmitter and the receiver. In this paper we assume CSI is available at both the ends in such a case, the beamforming vectors are obtained via SVD of the channels then the SVD of channel can be written as

$$
H=U \Sigma V^{H}
$$

where $U$ and $V$ are the two unitary matrices of size $M_{t} \times M_{t}$ and $M_{r}$ $\times M_{r}$ respectively, and $(.)^{H}$ denotes the conjugate transpose and $\sum$ is the $M_{t} \times M_{r}$ diagonal matrix with non-negative real numbers on the diagonal, $\sum=\operatorname{diag}\left(\lambda_{1}, \lambda_{2} \ldots \lambda_{M r}\right)$ where $\lambda_{1} \geq \ldots \ldots \geq \lambda_{M r}>0$ are the singular values and by using SVD, the MIMO channel is divided into independent and parallel sub channels.

\section{A. Proposed SVD-based Single Beamforming}

Only one symbol is transmitted over the subchannel with the largest gain. The channel matrix $H$ may be decomposed into a number of independent- orthogonal modes of excitation, which we will refer to as an eigenmodes of the channel.

The transmitter multiplies $x$ with $V$ before sending into the antennas and receiver multiplies the signals received on each antenna by the matrix $U^{H}$. Fig. 2, present the transmitter precoding and the receiver shaping transform the MIMO channel into $R_{H}$ (rank of $H$ ) parallel SISO channel with input $\bar{x}$ and output $\bar{y}$, since the overall transmission relationship

$$
\begin{aligned}
\bar{y} & =U^{H}(H x+n) \\
& =U^{H}\left(U \Sigma V^{H} x+n\right) \\
& =U^{H} U \Sigma V V^{H} \bar{x}+U^{H} n \\
\bar{y} & =\sum \bar{x}+\bar{n}
\end{aligned}
$$

where $\bar{n}=U^{H} n$, is the multiplication by a unitary matrix does not change the distribution of the noise (i.e.) $n$ and $\bar{n}$ they are identically distributed. The optimal vectors to be used at the transmitter side and receiver side are the first column of $U$ and $V$ corresponding to the largest singular value of $H$. Then, the received signal can be represented by

$$
\begin{aligned}
y & =\bar{x} U_{1}^{H} H V_{1}+U_{1}^{H} n \\
y & =\lambda_{1} \bar{x}+\bar{n}
\end{aligned}
$$

where $\lambda_{I}$ is the largest singular value of $H$. If the noise process is band limited with bandwidth $B$, the noise power of $n$ is given by $B N_{0}=2 \sigma_{n}{ }^{2}$. The equalized signal $y$ outlines that each transmitted symbol is weighted by its singular value which is shown from (9)

$$
y_{i}=\lambda_{1_{i}} \bar{x}_{i}+\overline{n_{i}} .
$$

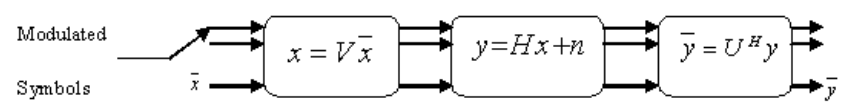

Figure 2. Schematic diagram of a transmit precoding and receive shaping scheme 
where, $i=1,2 \ldots \ldots M_{t}$ from (9) it is feasible to calculate each SISO SNR value shown by (10)

$$
S N R_{i}=\lambda_{1_{i}}^{2} \frac{\left|x_{i}\right|^{2}}{2 \sigma_{n}^{2}} .
$$

Since for a reliable communication the singular values must be greater than zero, therefore the singular values are also treated as MIMO processing gain, if the power of all symbols is normalized, so that the overall average power is given by

$$
\overline{p_{s}}=\frac{1}{M_{t}} \sum_{i=1}^{M_{t}}\left|x_{i}\right|^{2}=1 \text {. }
$$

\section{B. SVD-based multiple Beamforming}

Multiple symbols are simultaneously sent over different parallel subchannel. The optimal vectors to be used as weights at the transmitter side and receiver side are the first $S$ columns of $U$ and $V$ corresponding to the first $S$ larger singular values of $H$, when the $s$ subchannel for multiple beamforming becomes

$$
y_{k}=\frac{1}{\sqrt{S}} \lambda_{i} x_{i}+n_{i}
$$

Multiple beamforming achieves the diversity order of $\left(M_{t}-S+1\right)$ $\left(M_{r} S+1\right)$, for arbitrary $M_{t}, M_{r}, S$ where $S$ is the number of subchannel.

\section{MIMO SYSTEM CAPACITY}

The capacity of MIMO channel is defined as [10]

$$
C=\max _{f(x)} I(x, y)
$$

Where $f(s)$ is the probability distribution of the vector $s$ and $I(x, y)$ is the mutual information between vector $x$ and $y$. Therefore,

$I(x, y)$ is

$$
I(x, y)=\log _{2} \operatorname{det}\left(I_{M_{r}}+\frac{E_{x}}{M_{t} N_{0}} H R_{x x} H^{H}\right) b p s / H z
$$

where $E_{x}$ is the power across the transmitter irrespective of the number of transmit antennas $M_{t}$ and $I_{M r}$ is an $M_{t} \times M_{r}$ identity matrix, $N_{0}$ is a noise power and $R_{x x}$ is a covariance matrix of transmitted signal vector $x$ and from (13) and (14) capacity of MIMO channel is given by

$$
C=\max _{T r\left(R_{s s}\right)=M_{t}} \log _{2} \operatorname{det}\left(I_{M_{r}}+\frac{E_{x}}{M_{t} N_{0}} H R_{x x} H^{H}\right) b p s / H z
$$

$C$ is also called as error-free spectral efficiency or date rate per unit bandwidth that can be sustained reliably over the MIMO link. If the channel is unknown to the transmitter then the vector $x$ is statistically independent (i.e. $R_{x x=} I_{M t}$ ). This implies that the signals are independent and the power is equally divided among the transmit antenna. The capacity in such a case is

$$
C=\log _{2} \operatorname{det}\left(I_{M_{r}}+\frac{\rho}{N_{t}} H H^{H}\right)
$$

where $\rho=E_{x} / N_{0}$ is the average SNR at each receiver branch for further analysis of the MIMO channel capacity given in (16) is possible by diagonalizing the product matrix $\mathrm{HH}^{\mathrm{H}}$ either by eigenvalue decomposition or singular value decomposition. Now $\mathrm{HH}^{\mathrm{H}}$ is a $M_{t} \times M_{r}$ positive semidefinite Harmitian matrix. The eigendecomposition of such a matrix is given by $Q^{\wedge} Q^{H}$ where $Q$ is $M_{r} \times M_{t}$ matrix is satisfying $Q^{H} Q=Q Q^{H}=I_{M r}$ and ${ }^{\wedge}=\operatorname{diag}\left(\lambda_{l}\right.$, $\lambda_{2} \ldots . \Lambda_{M r}$ ) with $\lambda_{i} \geq 0$. We assume that the Eigen values are ordered so that $\lambda i \geq \lambda_{i+1}$ Then

$$
\lambda_{i}=\left\{\begin{array}{c}
\sigma_{i}^{2}, \text { if } i=1,2 \ldots . . r \\
0, \text { if } i=r, r+2 \ldots . M_{r}
\end{array}\right.
$$

where $\sigma_{\mathrm{i}}$ the singular values are obtained as $\sum=\operatorname{diag}\left(\sigma_{1}, \sigma_{2} \ldots \sigma_{r}\right)$ from the SVD of $H=U \sum V^{H}$. Then the capacity of the MIMO channel is given by

$$
C=\log _{2} \operatorname{det}\left(I_{M_{r}}+\frac{E_{x}}{M_{t} N_{0}} Q \wedge Q^{H}\right)
$$

Since, $Q^{H} Q=I_{M r}(18)$ simplifies to

$$
C=\log _{2} \operatorname{det}\left(I_{M_{r}}+\frac{E_{x}}{M_{t} N_{0}} \lambda_{i}\right)
$$

where $r$ is the rank of the channel and $\lambda_{i}(i=1,2 \ldots r)$ are positive Eigen values of $\mathrm{HH}^{\mathrm{H}}$. (20) Express the capacity of the MIMO channel as a sum of capacities of $r$ SISO channel, each having a power gain of $\lambda_{i}$ and the transmit power of $F_{6}\left\{M_{t}\right.$. When the channel is known at the transmitter, the maximum capacities of a MIMO channel can be achieved by using the water-filling principle [10] on the transmit covariance matrix. The capacity of MIMO channel is the sum of the individual parallel SISO channel capacities as given by

$$
C=\sum_{i=1}^{r} \log _{2}\left(1+\frac{E_{x} \gamma_{i}}{M_{t} N_{0}} \lambda_{i}\right)
$$

where $\gamma_{i}=\in\left\{\left|s_{i}\right|^{2}\right\}(i=1,2 \ldots \ldots . r)$ is the transmit energy in the $i^{\text {th }}$ subchannel such that $\sum_{i=1}^{r} \gamma_{i}=M_{t}$. Clearly, with a reduced number of non-zero singular values in (19) and (20), the capacity of the MIMO channel will be reduced because of the rank deficient channel matrix. This is the situation when the signals arriving at the receivers are correlated. Even though a high channel rank is necessary to obtain high spectral efficiency on a MIMO channel, low correlation is not a guarantee of high capacity.

\section{PERFORMANCE ANALYSIS 4.1 Derivation of the average BER for the BPSK over the Rayleigh fading channel}

The average BER for the BPSK system in the presence of Rayleigh fading is considered in this section for fading channel, the conditional BER for BPSK is given in [11]. 


$$
P_{b}(\gamma)=\frac{1}{2} \operatorname{erfc}\left(\sqrt{\frac{E_{b}}{N_{0}}}\right),
$$

However in the presence of channel $h$, the effective bit energy to noise ratio is $|h|^{2} E_{b} / N_{0}$. So that bit error probability for a given value of $h$ is

$$
P_{b / h}(\gamma)=\frac{1}{2} \operatorname{erfc}(\sqrt{\gamma}) .
$$

where $\gamma=|h|^{2} E_{b} / N_{0}$ is the instantaneous SNR per bit of the received signal $\operatorname{erfc}($.$) is the complementary error function. In$ addition, the probability density function (PDF) of $\gamma$ for rayleigh fading channel is given by [11].

$$
P(\gamma)=\frac{1}{\gamma} e^{\frac{\bar{\gamma}}{\gamma}} \quad \gamma \geq 0
$$

where $\bar{\gamma}=E_{b} / N_{0}$, the average BER can then be obtained using (21) and (22)

$$
\begin{aligned}
& P_{b}=\int_{0}^{\infty} \frac{1}{2} \operatorname{erfc}(\sqrt{\gamma}) p(\gamma) d \gamma,
\end{aligned}
$$

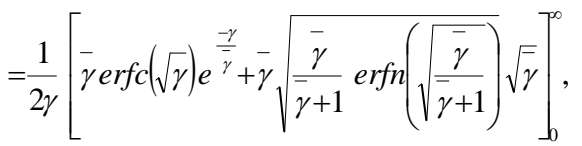

$$
\begin{aligned}
& =\frac{1}{2}\left(1-\frac{\bar{\gamma}}{\bar{\gamma}+1}\right) \text {, } \\
& P_{b}=0.5\left(1-\sqrt{\frac{E_{b} / N_{0}}{\left(E_{b} / N_{0}\right)+1}}\right) .
\end{aligned}
$$

\subsection{Derivation of the average SER for the M-QAM over the Rayleigh fading channel}

Let us consider square M-QAM signals whose constellation size is given by $M=2^{k}$ with $k$ even. The probability of symbol error for $\mathrm{M}-\mathrm{QAM}$ as in [12].

$$
\begin{aligned}
P_{s}(E) & =4\left(\frac{\sqrt{M}-1}{\sqrt{M}}\right) \times \\
& Q\left(\sqrt{\frac{3 E_{s}}{N_{0}(M-1)}}-4\left(\frac{\sqrt{M}-1}{\sqrt{M}}\right)^{2} Q^{2}\left(\sqrt{\frac{3 E_{s}}{N_{0}(M-1)}}\right)\right),
\end{aligned}
$$

where $Q(x)=\frac{1}{\pi} \int_{0}^{\frac{\pi}{2}} \exp \left(\frac{-x^{2}}{2 \sin ^{2} \theta}\right) d \theta$. is the Gaussian $Q$-function. The conditional SER for M-QAM with $L$-branch MRC receiver is given by (28) as

$$
\begin{aligned}
P_{s}\left(E /\left\{\gamma_{l}\right\}_{l=1}^{L}\right)= & 4\left(1-\frac{1}{\sqrt{M}}\right) Q\left(\sqrt{2 g_{Q A M}} \gamma_{t}\right) \\
& -4\left(1-\frac{1}{\sqrt{M}}\right)^{2} Q^{2}\left(\sqrt{2 g_{Q A M} \gamma_{t}}\right),
\end{aligned}
$$

where $\gamma_{l}=$ average SNR of $l^{\text {th }}$ branch and $\gamma_{t}=\sum_{l=1}^{L} \gamma_{L}$ is the total conditional SNR per symbol at the output the MRC and $g_{Q A M}=3 / 2[(M-1)]$, by averaging (28) over the Rayleigh pdf and using a standard integral involving the function $\mathrm{Q}^{2}($.$) , we$ obtain the performance of M-QAM over L i.i.d rayleigh fading channel as

$$
\begin{aligned}
P_{s}(E)= & 4\left(1-\frac{1}{\sqrt{M}}\right)\left(\frac{1-\mu_{c}}{2}\right)^{L} \sum_{l=0}^{L-N}\left(\begin{array}{c}
L-1+l \\
l
\end{array}\right)\left(\frac{1+\mu_{c}}{2}\right)^{l} \\
& -4\left(1-\frac{1}{\sqrt{m}}\right)^{2}\left(\frac{1}{4}-\frac{\mu_{c}}{\pi}\left(R_{b}-R_{a}\right)\right) .
\end{aligned}
$$

where

$$
\begin{gathered}
R_{b}=\left[\frac{\pi}{2}-\tan ^{-1} \mu_{c}\right] \sum_{l=0}^{L-1}\left[4\left(1+g_{Q A M} \bar{\gamma}\right)\right]^{l} \\
R_{a}=\sin \left(\tan ^{-1} \mu_{c}\right) \\
\times \sum_{l=1}^{L-1} \sum_{i=1}^{l} \frac{T_{i l}}{\left(1+g_{Q A M} \bar{\gamma}\right)^{l}}\left[\cos \left(\tan ^{-1} \mu_{c}\right)\right]^{2(l-i)+1} . \\
\mu_{c}=\sqrt{\frac{g_{Q A M} \bar{\gamma}}{1+g_{Q A M} \bar{\gamma}}}
\end{gathered}
$$

and

$T_{i l}=\frac{\left(\begin{array}{c}2 l \\ l\end{array}\right)}{\left(\begin{array}{c}2(l-i) \\ l-i\end{array}\right) 4^{i}(2(l-i)+1)}$.

\section{SIMULATION RESULTS}

In this section, we present the simulation results to demonstrate the BER of SVD-based single beamforming over the rayleigh fading channel. Here, we use three types of modulation techniques, one is BPSK and others are QPSK and 16-QAM. In that we compare the diversity performance of both modulations for single beamforming, with number of transmit and receive antennas. Fig. 3, we present the diversity performance of the SVD-based single beamforming with respect to $E_{b} / N_{0}$ in $d B$ with $M_{t}=M_{r}=2,3,4$ and BPSK. This figure shows that the BER of the SVD-based single beamforming is reduced with the increasing number of transmit and receive antennas. Fig. 4, displays the diversity performance of the SVD-based single beamforming with respect to $E_{b} / N_{0}$ in $d B$ with $M_{t}=M_{r}=2,3,4$ and QPSK. Fig. 5, displays the diversity performance of the SVD-based single beamforming with respect to $E_{b} / N_{0}$ in $d B$ with $M_{t}=M_{r}=2,3,4$ and 16-QAM. Both Fig.4 and 5, displays the same result as Fig.3. 
Fig.6, shows BER comparison for $2 \times 2$ single beamforming system with BPSK, QPSK and 16-QAM. This figure shows that the QPSK exhibit higher error rate than both BPSK and 16-QAM and, 16-QAM exhibit higher error rate than BPSK, however 16-QAM deliver higher raw data-rate than BPSK and QPSK. Fig.7 and 8, shows diversity performance comparison of the BPSK, QPSK and 16-QAM for SVD-based single beamforming with $M_{t}=M_{r}=2,3$. It shows that BER of the SVD-based single beamforming is reduced with the increasing number of transmit and receive antennas.

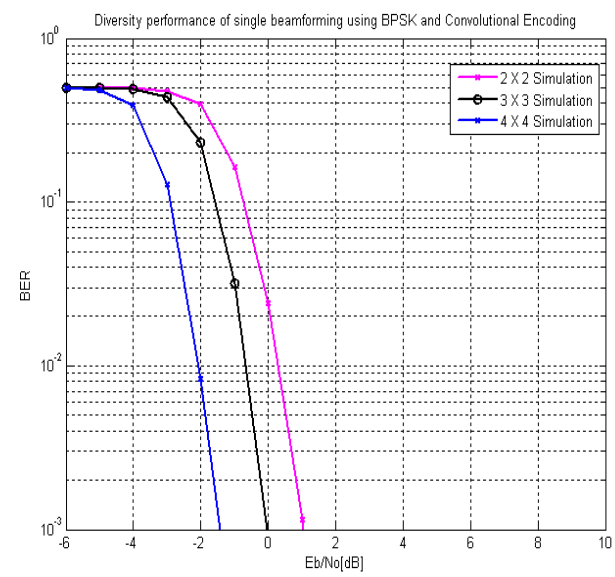

Figure 3. BER of SVD-based single beamforming for BPSK with $M_{t}=M_{r}=2,3,4$.

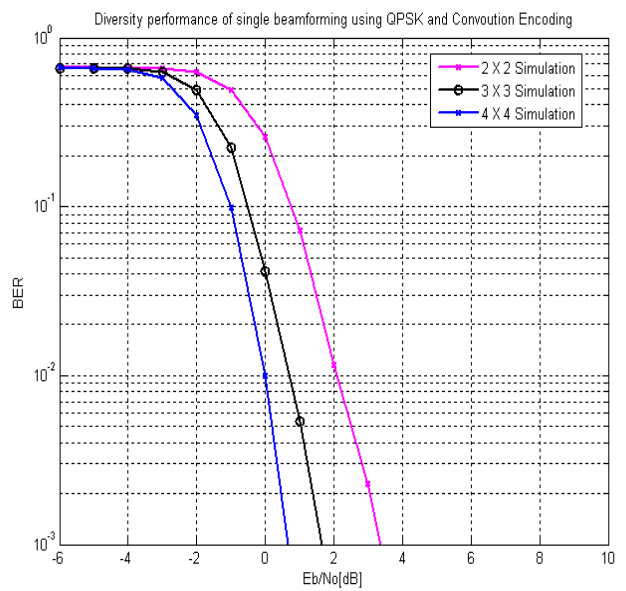

Figure 4. BER of SVD-based single beamforming for QPSK with $M_{t}=M_{r}=2,3,4$.

\section{CONCLUSION}

In this paper, it presented a transmit-beamforming scheme for MIMO system based on SVD-based single beamforming with BPSK, QPSK and 16-QAM modulations, and derived the expression for capacity of MIMO system when SVD-based beamforming is used for channel decomposition. It compares the BER performance of BPSK, QPSK and 16-QAM for single beamforming with different combination of transmitting and receiving antennas. Simulation results demonstrate that the proposed SVD-based beamforming schemes yield the better performance when compare to the other beamforming methods [13] with little bit of system complexity, when the increased number of transmit and receive antennas [13]. Also it shows that the BER performance of SVD-based beamforming with convolution encoding is reduced with the increased number of transmit and receive antennas and, the 16-QAM exhibit higher error rate than BPSK and QPSK; however 16-QAM delivers higher raw data-rate than BPSK and QPSK for various combination of transmit and receive antennas. This work can be extended by simulate the previous work with multiple beamforming (MBF) technique with SVD and also by using the generalized transmit maximum-ratio combining (G-TMRC) method for transmit beamforming to reducing complexity in SVD-based beamforming.

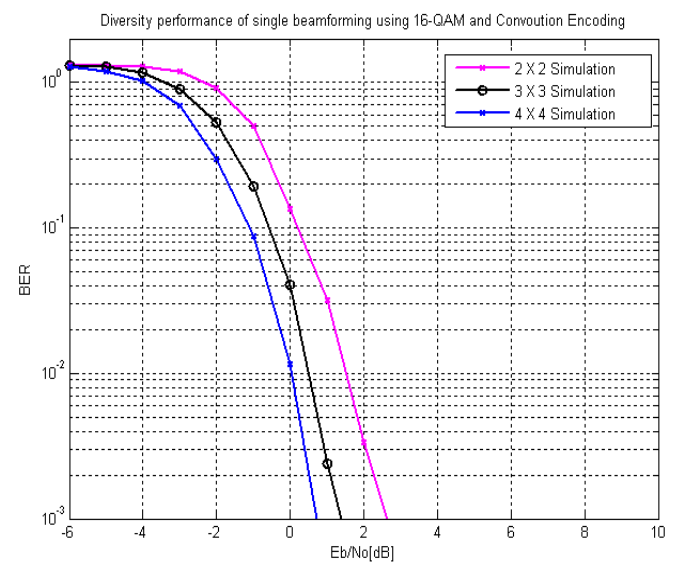

Figure 5. BER of SVD-based single beamforming for 16QAMwith $M_{t}=M_{r}=2,3,4$.

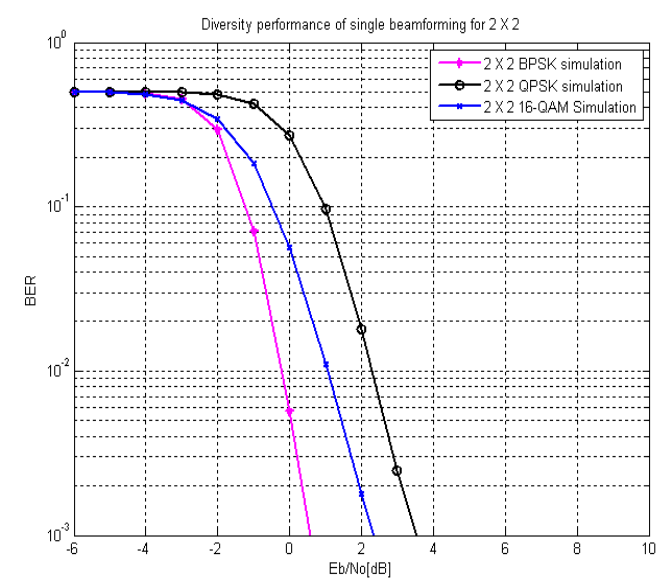

Figure 6. BER comparison of the BPSK, QPSK and 16-QAM for SVD-based single beamforming with $M_{t}=M_{r}=2$. 


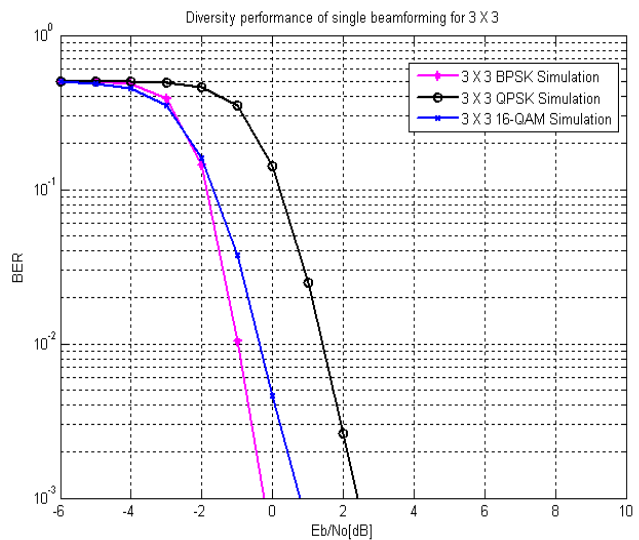

Figure 7. BER comparison of the BPSK, QPSK and 16-QAM for SVD-based single beamforming with $M_{t}=M_{r}=3$.

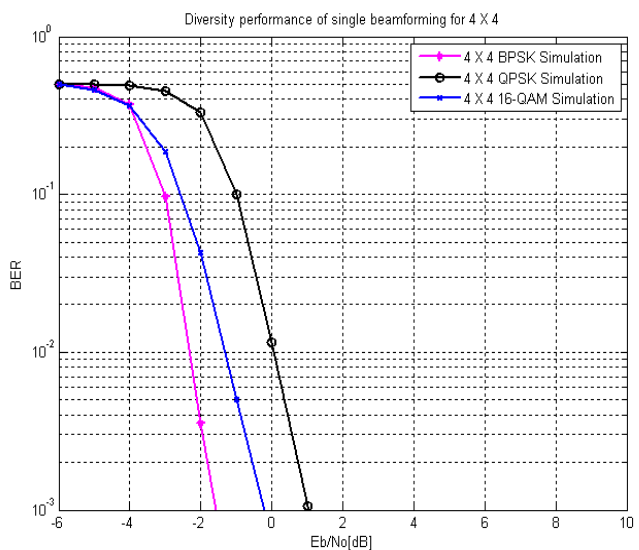

Figure 7. BER comparison of the BPSK, QPSK and 16-QAM for SVD-based single beamforming with $M_{t}=M_{r}=4$.

\section{REFERENCES}

[1] G. J. Foschini and M. J. Gans, "On limits of wireless communications in a fading environment when using multiple antennas," Wireless Personal Commun., vol. 6, pp. 311-335, Mar. 1998.

[2] I. E. Telatar, "Capacity of multi-antenna Gaussian channels," Eur. Trans. Telecom., vol. 10, pp. 585-595, Nov. 1999.

[3] . Paulraj and T. Kailath, "Increasing capacity in wireless broadcast systems using distributed transmission/directional reception (DTDR)”, Tech. Rep. U.S. Patent \#5345599, Sept 1994.

[4] V. Tarokh, N. Seshadri, and A. R. Calderbank, "Space-time codes for high data rate wireless communications: Performance criterion and code concstruction", IEEE Trans. Information Theory, vol. 44, no. 2, pp. 744-765, May 1998.

[5] A. J. Goldsmith and P. P. Varaiya, "Capacity of fading channels with channel side information," IEEE Trans. Inform. Theory, vol. 43, pp. 1986-1992, Nov. 1997.

[6] A. J. Goldsmith, S. A. Jafar, N. J. Jindal, and S. Vishwanath, "Capacity of limits of MIMO channels," IEEE J. Select. areas in communications, vol. 21, pp. 684-702, June 2003.

[7] D. P. Palomar, "A unified framework for communications through MIMO channels," Ph.D. dissertation, Universitat Politecnica de Catalunya, Barcelona, Spain, May 2003.

[8] G. Foschini and M. J. Gans, "Layered space-time architecture for wireless communication in a fading environment when using multielement antennas," Bell Labs Tech. J., vol. 1, no. 2, pp. 41-59, 1996.

[9] H. Sampath, P. Stoica, and A. Paulraj, "Generalized linear precoder and decoder design for MIMO channels using the weighted MMSE criterion," IEEE Trans. Commun., vol. 49, pp. 2198-2206, Dec. 2001.

[10] Mohinder jankiraman "Space-Time codes and MIMO system" Artech House Universal Personal Communication Series,2004.

[11] John G. Proakis "Digital Communication" $4^{\text {th }}$ edition, McGraw-Hill higher education, 2001.

[12] Marvin K.Simon and Mohamed-Slim Aluini "Digital Communication over Fading Channels" $2^{\text {nd }}$ edition, a John Wiley \& Sons,Inc.,Publication, 2005.

[13] S. Park, H. Lee, S-R Lee, I. Lee, "A New Beamforming Structure Based on Transmit-MRC for Closed-Loop MIMO Systems" IEEE Trans. Commun.,vol. 57, pp.1847-1856,june.2009. 